\title{
Behavior of a Scale Factor for Wiener Integrals of an Unbounded Function
}

\section{Young Sik Kim}

Department of Mathematics, College of Natural Sciences, Industry-University Cooperation Foundation, Hanyang University, Seoul, Republic of Korea

Email: yoskim@hanyang.ac.kr

How to cite this paper: Kim, Y.S. (2019) Behavior of a Scale Factor for Wiener Integrals of an Unbounded Function. Applied Mathematics, 10, 326-332.

https://doi.org/10.4236/am.2019.105023

Received: February 2, 2019

Accepted: May 20, 2019

Published: May 23, 2019

Copyright () 2019 by author(s) and Scientific Research Publishing Inc. This work is licensed under the Creative Commons Attribution International License (CC BY 4.0).

http://creativecommons.org/licenses/by/4.0/

\begin{abstract}
The purpose of this paper is to investigate the behavior of a scale factor for Wiener integrals about the unbounded function $F(x)=\exp \left\{a \sum_{j=1}^{n} \int_{0}^{T} \alpha_{j} \mathrm{~d} x\right\}$, where $\left\{\alpha_{1}, \alpha_{2}, \cdots, \alpha_{n}\right\}$ is an orthonormal set of elements in $L_{2}[0, T]$ on the Wiener space $C_{0}[0, T]$.

\section{Keywords}

Wiener Space, Wiener Integral, Analytic Wiener Integral, Analytic Feynman Integral, Scale Factor
\end{abstract}

\section{Introduction}

In [1], M. D. Brue introduced the functional transform on the Feynman integral (1972). In [2], R. H. Cameron wrote the paper about the translation pathology of a Wiener spac (1972). In [3] [4] [5], R. H. Cameron and W. T. Martin proved some theorems on the transformation and the translation and used the expression of the change of scale for Wiener integrals (1944, 1947). In [6] [7], R. H. Cameron and D. A. Storvick proved relationships between Wiener integrals and analytic Feynman integrals to prove the change of scale formula for Wiener integral on the Wiener space in 1987. In [8], M. D. Gaysinsky and M. S. Goldstein proved the Self-Adjointness of a Schrödinger Operator and Wiener Integrals (1992).

In [9], G. W. Johnson and M. L. Lapidus wrote the paper about the Feynman integral and Feynman's Operational Calculus (2000). In [10], G. W. Johnson and D. L. Skoug proved the scale-invariant measurability in Wiener Space (1979). 
In [11] and [12], Y. S. Kim proved a change of scale formula for Wiener integrals about cylinder functions $f\left(\left(h_{1} x\right)^{\sim}, \cdots,\left(h_{n}, x\right)^{\sim}\right)$ with $f \in L_{p}\left(R^{n}\right), 1 \leq p \leq \infty$ on the abstract Wiener space: the analytic Wiener integral exists for $f \in L_{p}\left(R^{n}\right), 1 \leq p \leq \infty$, and the analytic Feynman integral exists for $f \in L_{1}\left(R^{n}\right)$ (1998) and (2001). But the Feynman integral does not always exist for $1<p$.

In [13], Y. S. Kim investigates a behavior of a scale factor for the Wiener integral of a function $F(x)=\exp \left\{\int_{0}^{T} \theta(t, x(t)) \mathrm{d} t\right\}$, where $\theta:[0, T] \times R \rightarrow \mathbf{C}$ is defined by $\theta(t, u)=\int_{R} \exp \{i u v\} \mathrm{d} \sigma_{t}(v)$ which is a Fourier-Stieltzes transform of a complex Borel measure $\sigma_{t} \in \mathbf{M}(R)$ and $\mathbf{M}(R)$ is a set of complex Borel measures defined on $\mathrm{R}$.

In this paper, we investigate the behavior of a scale factor $\rho>0$ for the Wiener integral $\int_{C_{0}[0, T]} F(\rho x) \mathrm{d} m(x)$ which is defined on the Wiener space $C_{0}[0, T]$ about the unbounded function $F(x)=\exp \left\{a \sum_{j=1}^{n} \int_{0}^{T} \alpha_{j} \mathrm{~d} x\right\}$ with $a>0$, where $\left\{\alpha_{1}, \alpha_{2}, \cdots, \alpha_{n}\right\}$ is an orthonormal set of elements in $L_{2}[0, T]$ on the Wiener space $C_{0}[0, T]$.

\section{Definitions and Preliminaries}

Let $C_{0}[0, T]$ denote the space of real-valued continuous functions $x$ on $[0, T]$ such that $x(0)=0$. Let $\mathcal{M}$ denote the class of all Wiener measurable subsets of $C_{0}[0, T]$ and let $m$ denote a Wiener measure and $\left(C_{0}[0, T], \mathcal{M}, m\right)$ be a Wiener measure space and we denote the Wiener integral of a function $F: C_{0}[0, T] \rightarrow \mathbf{C}$ by $\int_{C_{0}[0, T]} F(x) \mathrm{d} m(x)$.

A subset $E$ of $C_{0}[0, T]$ is said to be scale-invariant measurable if $\rho E \in \mathcal{M}$ for each $\rho>0$, and a scale-invariant measurable set $N$ is said to be scale-invariant null if $m(\rho N)=0$ for each $\rho>0$. A property that holds except on a scale-invariant null set is said to hold scale-invariant almost everywhere (s-a.e.). If two functionals $F$ and $G$ are equal s-a.e., we write $F \approx G$. A function $F$ defined on the scale invariant measurable set $E$ is a scale invariant measurable function if $F(\alpha x)$ is a Wiener measurable function for all $\alpha>0$.

Throughout this paper, let $\mathbf{R}^{n}$ denote the $n$-dimensional Euclidean space and let $\mathbf{C}, \mathbf{C}_{+}$, and $\mathbf{C}_{+}^{\sim}$ denote the set of complex numbers, the set of complex numbers with positive real part, and the set of non-zero complex numbers with nonnegative real part, respectively.

Definition 2.1. Let $F$ be a complex-valued measurable function on $C_{0}[0, T]$ such that the integral

$$
J(F ; \lambda)=\int_{C_{0}[0, T]} F\left(\lambda^{-\frac{1}{2}} x\right) \mathrm{d} m(x)
$$

exists for all real $\lambda>0$. If there exists a function $J^{*}(F ; z)$ analytic on $\mathbf{C}_{+}$ such that $J^{*}(F ; \lambda)=J(F ; \lambda)$ for all real $\lambda>0$, then we define $J^{*}(F ; z)$ to be the analytic Wiener integral of $F$ over $C_{0}[0, T]$ with parameter $z$, and for 
each $z \in \mathbf{C}_{+}$, we write

$$
I^{a w}(F ; z)=J^{*}(F ; z) \equiv \int_{C_{0}[0, T]}^{a n w_{z}} F(x) \mathrm{d} m(x) .
$$

Let $q$ be a non-zero real number and let $F$ be a function defined on $C_{0}[0, T]$ whose analytic Wiener integral exists for each $z$ in $\mathbf{C}_{+}$. If the following limit exists, then we call it the analytic Feynman integral of $F$ over $C_{0}[0, T]$ with parameter $q$, and we write

$$
I^{a f}(F ; q)=\lim _{z \rightarrow-i q} I^{a w}(F ; z) \equiv \int_{C_{0}[0, T]}^{a n w_{q}} F(x) \mathrm{d} m(x),
$$

where $z$ approaches $-i q$ through $\mathbf{C}_{+}$and $i^{2}=-1$.

Let $\left\{e_{k}\right\}_{k=1}^{n}$ be a complete orthonormal set and $e_{k} \in C[0, T] \cap B[0, T]$ for $k=1,2, \cdots, n$ and $\alpha \in L_{2}[0, T]$ and $x \in C_{0}[0, T]$. We define a Paley-Wiener-Zygmund integral (P.W.Z) of $x$ with respect to $\alpha$ by

$$
\int_{0}^{T} \alpha(t) \mathrm{d} x(t) \equiv \lim _{n \rightarrow \infty} \int_{0}^{T} \sum_{k=1}^{n}\left(\alpha, e_{k}\right) e_{k}(t) \mathrm{d} x(t) .
$$

Theorem 2.2 (Wiener Integration Formula). Let $C_{0}[0, T]$ be a Wiener space. Then

$$
\begin{aligned}
& \int_{C_{0}[0, T]} f\left(\int_{0}^{T} \alpha_{1} \mathrm{~d} x, \int_{0}^{T} \alpha_{2} \mathrm{~d} x, \cdots, \int_{0}^{T} \alpha_{n} \mathrm{~d} x\right) \mathrm{d} m(x) \\
& =\left(\frac{1}{2 \pi}\right)^{\frac{n}{2}} \int_{\mathbf{R}^{n}} f(\vec{u}) \exp \left\{-\frac{1}{2} \sum_{j=1}^{n} u_{j}^{2}\right\} \mathrm{d} \vec{u}
\end{aligned}
$$

where $\left\{\alpha_{1}, \alpha_{2}, \cdots, \alpha_{n}\right\}$ is an orthonormal set of elements in $L_{2}[0, T]$ and $f: \mathbf{R}^{n} \rightarrow \mathbf{C}$ is a Lebesgue measurable function and $\vec{u}=\left(u_{1}, u_{2}, \cdots, u_{n}\right)$ and $\mathrm{d} \vec{u}=\mathrm{d} u_{1} \mathrm{~d} u_{2} \cdots \mathrm{d} u_{n}$ and $\int_{0}^{T} \alpha_{j} \mathrm{~d} x$ is a Paley-Wiener-Zygmund integral for $1 \leq j \leq n$.

Remark. We will use several times the following well-known integration formula:

$$
\int_{\mathbf{R}} \exp \left\{-a u^{2}+i b u\right\} \mathrm{d} u=\sqrt{\frac{\pi}{a}} \exp \left\{-\frac{b^{2}}{4 a}\right\}
$$

where $a$ is a complex number with $\operatorname{Re} a>0, b$ is a real number, and $i^{2}=-1$.

\section{Main Results}

Define a function $F: C_{0}[0, T] \rightarrow \mathbf{C}$ on the Wiener space by

$$
F(x)=\exp \left\{a \sum_{j=1}^{n} \int_{0}^{T} \alpha_{j} \mathrm{~d} x\right\}
$$

where $a>0$ is a finite real number and $\left\{\alpha_{1}, \alpha_{2}, \cdots, \alpha_{n}\right\}$ is an orthonormal set of elements in $L_{2}[0, T]$.

Lemma 3.1. For a finite real number $a>0$, the unbounded cylinder function $F(x)$ in (3.1) is a Wiener integrable function.

Proof. By the Wiener integration Formula (2.4), we have that for a finite real 
number $a>0$

$$
\begin{aligned}
& \int_{C_{0}[0, T]} F(x) \mathrm{d} m(x) \\
& =\left(\frac{1}{2 \pi}\right)^{\frac{n}{2}} \cdot \int_{R^{n}} \exp \left\{a \sum_{j=1}^{n} u_{j}-\frac{1}{2} \sum_{j=1}^{n} u_{j}^{2}\right\} \mathrm{d} \vec{u} \\
& =\left(\frac{1}{2 \pi}\right)^{\frac{n}{2}} \cdot(2 \pi)^{\frac{n}{2}} \cdot \exp \left\{+\frac{n}{2} a^{2}\right\} \\
& =\exp \left\{+\frac{n}{2} a^{2}\right\}<\infty
\end{aligned}
$$

Remark. If we let $F(x)=f\left(\int_{0}^{T} \alpha_{1} \mathrm{~d} x, \int_{0}^{T} \alpha_{2} \mathrm{~d} x, \cdots, \int_{0}^{T} \alpha_{n} \mathrm{~d} x\right)$ and $f: R^{n} \rightarrow \mathbf{C}$, then $f(\vec{u})=\exp \left\{a \sum_{j=1}^{n} u_{j}\right\}$ is unbounded for a finite real number $a>0$.

Lemma 3.2. Let $F: C_{0}[0, T] \rightarrow \mathbf{C}$ be defined by (3.1). For a finite real $\rho>0$ and a finite real $a>0$,

$$
\int_{C_{0}[0, T]} F(\rho x) \mathrm{d} m(x)=\exp \left\{+\frac{n}{2} a^{2} \rho^{2}\right\}
$$

Proof. By the Wiener integration Formula (2.4), we have that

$$
\begin{aligned}
& \int_{C_{0}[0, T]} F(\rho x) \mathrm{d} m(x) \\
& =\left(\frac{1}{2 \pi}\right)^{\frac{n}{2}} \cdot \int_{R^{n}} \exp \left\{a \rho \sum_{j=1}^{n} u_{j}-\frac{1}{2} \sum_{j=1}^{n} u_{j}^{2}\right\} \mathrm{d} \vec{u} \\
& =\left(\frac{1}{2 \pi}\right)^{\frac{n}{2}} \cdot(2 \pi)^{\frac{n}{2}} \cdot \exp \left\{+\frac{n}{2} a^{2} \rho^{2}\right\} \\
& =\exp \left\{+\frac{n}{2} a^{2} \rho^{2}\right\}<\infty
\end{aligned}
$$

Lemma 3.3. Let $F: C_{0}[0, T] \rightarrow \mathbf{C}$ be defined by (3.1). For a finite real $\rho>0$ and a finte real $a>0$,

$$
\int_{C_{0}[0, T]} F(\rho x) \mathrm{d} m(x)=\left[\int_{C_{0}[0, T]} F(x) \mathrm{d} m(x)\right]^{\rho^{2}}
$$

Proof. By the above Lemma, we have that

$$
\begin{aligned}
& \int_{C_{0}[0, T]} F(\rho x) \mathrm{d} m(x) \\
& =\exp \left\{+\frac{n}{2} a^{2} \rho^{2}\right\} \\
& =\left[\exp \left\{+\frac{n}{2} a^{2}\right\}\right]^{\rho^{2}} \\
& =\left[\int_{C_{0}[0, T]} F(x) \mathrm{d} m(x)\right]^{\rho^{2}}
\end{aligned}
$$

Now we define a concept of the scale factor for the Wiener integral which was first defined in [13]:

Definition 3.4. We define the scale factor for the Wiener integral by the 
real number $\rho>0$ of the absolute value of the Wiener integral:

$$
G(\rho)=\left|\int_{C_{0}[0, T]} F(\rho x) \mathrm{d} m(x)\right|
$$

where $G: R \rightarrow \mathbf{R}$ is a real valued function defined on $R$.

Property. <Behavior of a scale factor for the Wiener integral.>

We investigate the interesting behavior of the scale factor for the Wiener integral by analyzing the Wiener integral as followings: For real $\rho>0$ and for a finite real number $a>0$,

$$
\int_{C_{0}[0, T]} F(\rho x) \mathrm{d} m(x)=\exp \left\{+\frac{n}{2} a^{2} \rho^{2}\right\} .
$$

Example. For the scale factor $\rho=\left\{\cdots, \frac{1}{100}, \frac{1}{10}, 1,10,10^{2}, \cdots\right\}$, we can investigate the very interesting behavior of the Wiener integral:

$$
\begin{aligned}
& \text { (a) } \int_{C_{0}[0, T]} F\left(\frac{1}{100} x\right) \mathrm{d} m(x)=\left[\int_{C_{0}[0, T]} F(x) \mathrm{d} m(x)\right]^{\frac{1}{10000}} \\
& \text { (b) } \int_{C_{0}[0, T]} F\left(\frac{1}{10} x\right) \mathrm{d} m(x)=\left[\int_{C_{0}[0, T]} F(x) \mathrm{d} m(x)\right]^{\frac{1}{100}} \\
& \text { (c) }\left[\int_{C_{0}[0, T]} F\left(\frac{1}{100} x\right) \mathrm{d} m(x)\right]^{100}=\left[\int_{C_{0}[0, T]} F\left(\frac{1}{10} x\right) \mathrm{d} m(x)\right] \\
& \text { (d) } \int_{C_{0}[0, T]} F(\rho x) \mathrm{d} m(x)=\left[\int_{C_{0}[0, T]} F(x) \mathrm{d} m(x)\right]^{\rho^{2}}
\end{aligned}
$$

Remark. <Interpretation of a scale factor for Wiener integrals of an unbounded cylinder function.>

1) Whenever the scale factor $\rho>1$ is increasing, the Wiener integral increases very rapidly. Whenever the scale factor $0<\rho<1$ is decreasing, the Wiener integral decreases very rapidly.

2) The function $G(\rho)=\left|\int_{C_{0}[0, T]} F(\rho x) \mathrm{d} m(x)\right|$ for $F(x)$ in (3.1) is an increasing function of a scale factor $\rho>0$, because the exponential function $y=\mathrm{e}^{x^{2}}$ is an increasing function of $x \in R$.

3) Whenever the scale factor $\rho>0$ is increasing and decreasing, the Wiener integral varies very rapidly.

\section{Conclusions}

What we have done in this research is that we investigate the very interesting behavior of the scale factor for the Wiener integral of an unbounded function.

From these results, we find an interesting property for the Wiener integral as a function of a scale factor which was first defined in [13].

Note that the function in [13] is bounded and the function of this paper is unbounded!

Finally, we introduce the motivation and the application of the Wiener 
integral and the Feynman integral and the relationship between the scale factor and the heat (or diffusion) equation:

\section{Remark.}

1) The solution of the heat (or diffusion) equation

$$
\frac{\partial \psi}{\partial t}=-\frac{i}{h}\left[-\frac{h^{2}}{2 m} \frac{\partial \psi^{2}}{\partial \xi^{2}}+V(\xi) \psi\right]
$$

is that for a real $\lambda>0$,

$$
\psi_{\lambda}(t, \xi)=\int_{C_{0}^{t}} \exp \left\{-\frac{i}{h} \int_{0}^{t} V\left(\lambda^{-\frac{1}{2}} x(s)+\xi\right) \mathrm{d} s\right\} \cdot \psi\left(\lambda^{-\frac{1}{2}} x(s)+\xi\right) \mathrm{d} m(s)
$$

where $\psi_{\lambda}(\cdot, \xi)=\phi(\xi)$ and $\phi \in L_{2}\left(R^{d}\right)$ and $\xi \in R^{d}$ and $x(\cdot)$ is a $R^{d}$ -valued continuous function defined on $[0, t]$ such that $x(0)=0$.

2) $H=-\Delta+V$ is the energy operator (or, Hamiltonian) and $\Delta$ is a Laplacian and $V: R^{d} \rightarrow R$ is a potential. This Formula (3.11) is called the Feynman-Kac formula. The application of the Feynman-Kac Formula (in various settings) has been given in the area: diffusion equations, the spectral theory of the schrödinger operator, quantum mechanics, statistical physics, for more details, see the paper [8] and the book [12].

3) If we let $\lambda=\rho^{-2}$, the solution of this heat (or diffusion) equation is

$$
\psi_{\rho}(t, \xi)=\int_{C_{0}^{t}} \exp \left\{-\frac{i}{h} \int_{0}^{t} V(\rho x(s)+\xi) \mathrm{d} s\right\} \cdot \phi(\rho x(s)+\xi) \mathrm{d} m(s)
$$

4) If we let $h=\frac{m}{i \lambda}=-i m \rho^{-2}$, then

$$
\psi_{\rho}(t, \xi)=\int_{C_{0}^{t}} \exp \left\{+m \rho^{2} \int_{0}^{t} V(\rho x(s)+\xi) \mathrm{d} s\right\} \cdot \phi(\rho x(s)+\xi) \mathrm{d} m(s)
$$

is a solution of a heat (or diffusion) equation:

$$
\frac{\partial \psi}{\partial t}=\frac{1}{m \rho^{2}}\left[\left(\frac{m^{2} \rho^{2}}{2 m}\right) \frac{\partial \psi^{2}}{\partial \xi^{2}}+V(\xi) \psi\right] .
$$

This equation is of the form:

$$
\frac{\partial \psi}{\partial t}=\frac{1}{2} \frac{\partial \psi^{2}}{\partial \xi^{2}}+\frac{1}{m \rho^{2}} V(\xi) \psi
$$

5) If we let $F(x)=\exp \left\{-\frac{i}{h} \int_{0}^{t} V\left(\lambda^{-\frac{1}{2}} x(s)+\xi\right) \mathrm{d} s \cdot \phi\left(\lambda^{-\frac{1}{2}} x(s)+\xi\right)\right\}$, then we can express the solution of the heat (or diffusion) equation by the formula

$$
\psi_{\rho}(t, \xi)=\int_{C_{0}^{t}} F(\rho x) \mathrm{d} m(x), \psi_{\lambda}(t, \xi)=\int_{C_{0}^{t}} F\left(\lambda^{-\frac{1}{2}} x\right) \mathrm{d} m(x)
$$

6) By this motivation, we first define the scale factor of the Wiener integral by the real number $\rho>0$ in the paper [13].

Remark. $<$ Gratitude for the Refree $>$ I am very grateful for the referee to comment in details. 


\section{Supported}

This article was supported by the National Research Foundation grant NRF-2017R1A6311030667.

\section{Conflicts of Interest}

The author declares no conflicts of interest regarding the publication of this paper.

\section{References}

[1] Brue, M.D. (1972) A Functional Transform for Feynman Integrals Similar to Fourier Transform. Thesis, University of Minnesota.

[2] Cameron, R.H. (1954) The Translation Pathology of Wiener Space. Duke Mathematical Journal, 21, 623-628. https://doi.org/10.1215/S0012-7094-54-02165-1

[3] Cameron, R.H. and Martin, W.T. (1944) On Transformations of Wiener Integrals under Translations. Annals of Mathematics, 45, 386-396. https://doi.org/10.2307/1969276

[4] Cameron, R.H. and Martin, W.T. (1945) Transformations for Wiener Integrals under a General Class of Linear Transformations. Transactions of the American Mathematical Society, 58, 184-219. https://doi.org/10.1090/S0002-9947-1945-0013240-1

[5] Cameron, R.H. and Martin, W.T. (1947) The Behavior of Measure and Measurability under Change of Scale in Wiener Space. Bull. Transactions of the American Mathematical Society, 53, 130-137. https://doi.org/10.1090/S0002-9904-1947-08762-0

[6] Cameron, R.H. and Storvick, D.A. (1987) Relationships between the Wiener Integral and the Analytic Feynman Integral. Supplemento ai Rendiconti del Circolo Matematico di Palermo, Serie II-numero, 17, 117-133.

[7] Cameron, R.H. and Storvick, D.A. (1987) Change of Scale Formulas for Wiener Integral. Supplemento ai Rendiconti del Circolo Matematico di Palermo, Serie II-numero, 17, 105-115.

[8] Gaysinsky, M.D. and Goldstein, M.S. (1992) Self-Adjointness of Schrödinger Operator and Wiener Integrals. Integral Equations and Operator Theory, 15, 973-990. https://doi.org/10.1007/BF01203123

[9] Johnson, G.W. and Lapidus, M.L. (2000) The Feynman Integral and Feynman's Operational Calculus. Oxford Science Publications, Oxford.

[10] Johnson, G.W. and Skoug, D.L. (1979) Scale-Invariant Measurability in Wiener Space. Pacific Journal of Mathematics, 283, 157-176. https://doi,org/10.2140/pjm.1979.83.157

[11] Kim, Y.S. (1998) A Change of Scale Formula for Wiener Integrals of Cylinder Functions on the Abstract Wiener Space. International Journal of Mathematics and Mathematical Sciences, 21, 73-78. https://doi.org/10.1155/S0161171201004537

[12] Kim, Y.S. (2001) A Change of Scale Formula for Wiener Integrals of Cylinder Functions on the Abstract Wiener Space II. International Journal of Mathematics and Mathematical Sciences, 25, 231-237. https://doi.org/10.1155/S0161171201004537

[13] Kim, Y.S. (2018) Behavior of a Scale Factor for Wiener Integrals and a Fourier-Stieltzes Transform on the Wiener Space. Applied Mathematics, 9, 488-495. https://doi.org/10.4236/am.2018.95035 